ANL-6551

Physics

(TID-4500, 18th Ed.)

AEC Research and

Development Report

ARGONNE NATIONAL LABORATORY

9700 South Cass Avenue

Argonne, Illinois

\title{
A PROCEDURE FOR THE EVALUATION OF NEUTRON-SCATTERING CROSS SECTION IN THE INCOHERENT APPROXIMATION \\ by
}

V. Z. Jankus

Reactor Engineering Division

September 1962

Operated by The University of Chicago under

Contract W-31-109-eng-38 


\section{DISCLAIMER}

This report was prepared as an account of work sponsored by an agency of the United States Government. Neither the United States Government nor any agency Thereof, nor any of their employees, makes any warranty, express or implied, or assumes any legal liability or responsibility for the accuracy, completeness, or usefulness of any information, apparatus, product, or process disclosed, or represents that its use would not infringe privately owned rights. Reference herein to any specific commercial product, process, or service by trade name, trademark, manufacturer, or otherwise does not necessarily constitute or imply its endorsement, recommendation, or favoring by the United States Government or any agency thereof. The views and opinions of authors expressed herein do not necessarily state or reflect those of the United States Government or any agency thereof. 


\section{DISCLAIMER}

Portions of this document may be illegible in electronic image products. Images are produced from the best available original document. 
TABLE OF CONTENTS

Page

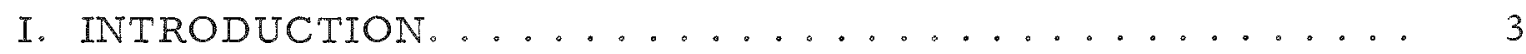

II. INITIAL CALCULATION OF SEVERAL PARAMETERS . . . . . 5

III. THE MULTIPHONON EXPANSION. . . . . . . . . . . . . 9

IV. CALCULATION OF DIFFERENTIAL SCATTERING CROSS SECTION AND SCATTERING KERNEL. ........... II

V. CALCULATION OF MULTIGROUP INELASTIC MATRICES AND TRANSPORT CROSS SECTIONS ............ 12

A. Calculation by Multiphonon Expansion ......... 13

B. Calculation by an Asymptotic Formula.......... 14

C. Integration over Final Energies and Averaging over Initial Energies .................. 17

APPENDICES

A. Evaluation of $c_{y}^{(n)} \ldots \ldots \ldots 19$

B. The Steepest Descent Method and Convergence of Multiphonon Expansion ............... 23

C. Averaging over Directions of Polarization for

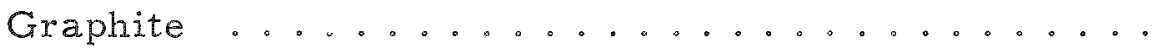

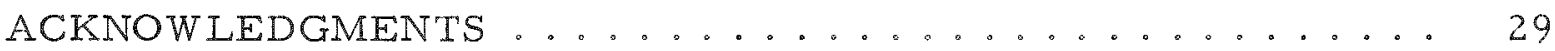

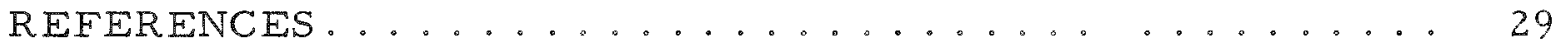




\title{
A PROCEDURE FOR THE EVALUATION OF NEUTRON-SCATTERING CROSS SECTION IN THE INCOHERENT APPROXIMATION
}

by

V. Z. Jankus

\begin{abstract}
Using some simplifying as sumptions about the details of phononfrequency distribution, we evaluated exactly the contributions of 25 phonons. By means of these contributions, the differential scattering cross section $\sigma\left(\mathrm{E} \rightarrow \mathrm{E}^{\prime}, \theta\right)$, the scattering law $\mathrm{S}(\alpha, \beta)$, or inelastic-scattering matrices and transport cross sections for multigroup calculations may be calculated. In the evaluation of multigroup parameters use is made of an asymptotic expression when the phonon expansions fail to converge.
\end{abstract}

When the phonon-frequency distribution is considered dependent on the direction of polarization (as in graphite), then averages over all directions are obtained.

\section{INTRODUCTION}

The basic ideas underlying present scheme of evaluation of the inelastic neutron cross section have been explained briefly in a paper presented at a meeting of the American Nuclear Society.(1) This report is intended to complete the presentation of the procedure, which is oriented towards computer application. Thus, in addition to a brief derivation of mathematical formulae, this report includes descriptions of calculational procedures which may be used with a computing machine. Some meas ures taken are obviously somewhat arbitrary and have been included in this description for the sake of completeness.

Essentially, we propose to evaluate the inelastic-scattering cross section in the incoherent approximation for a simple cubic Bravais lattice. The main formula $(2)$ does not depend on the polarization of the phonons.

The basic part of the procedure (see Chapter III) is the evaluation of repeated convolutions to obtain contributions due to 25 phonons. To avoid errors in multiple integrations, it is assumed initially that the phononfrequency distribution $\rho(\omega)$ is such that

$$
f(\omega)=\frac{\rho(\omega)}{\omega s h(\omega / 2 k T)}
$$


can be represented by a broken line with break points at integral multiples of $\Delta \omega$. (Usually $\Delta \omega$ is much smaller than $\mathrm{kT}$.) Then the multiphonon contributions are determined exactly by a simple procedure [see Eqs. (16) and (17)].

Having the multiphonon contributions we can calculate differential scattering cross sections $\sigma\left(E \rightarrow E^{\prime}, \theta\right)$, the scattering law $S(\alpha, \beta)$, or inelastic scattering matrices and transport cross sections to be used in multigroup calculations. In all these cases it is assumed that contributions of neglected phonons decrease in a geometric progression. A correction term is added if it is smaller than $10 \%$ of the total. Otherwise, it must be considered that the expansion using only 25 phonons is unsatisfactory for the determination of $\sigma\left(\mathrm{E}_{\rightarrow} \mathrm{E}^{\prime}, \theta\right)$ and $S(\alpha, \beta)$. Fortunately, this is unlikely for experimentally observable energy and momentum transfers.

In the evaluation of multigroup scattering matrices, integration over scattering angle $\theta$ has been performed analytically, and $\sigma\left(E \rightarrow E^{\prime}\right)$ is computed by use of the multiphonon expansion if it converges satisfactorily: otherwise, an asymptotic expression has been chosen to fit the region of drop-off of the inelastic cross section at high energies, $\left|E-E^{\prime}\right| \approx$ $\mu\left(\sqrt{E}+\sqrt{E^{3}}\right)^{2}$. It is not good for much larger energy transfers. How ever, the inelastic cross section in that region is very small, and thus the error is not expected to affect the subsequent flux calculations. In the instances in which the energy change is finite but the incident energy is very large, the asymptotic expansion may not be good, but in this case the multiphonon expansion still converges (provided $n_{\max } \geqq 4 a_{2} g(0)$, as seen in Appendix B). In any case, the value of this nearly elastic cross section is not expected to effect great changes in the reactor flux.

Similar asymptotic expressions have been used previously by Schofield and Hassit $(2)$ and by Sjolander. (3) However, they have used this approach to evaluate individual multiphonon contributions. In our procedure this expansion is used for the main formula as a whole, thus saving an appreciable amount of computation.

Although in our main formula it is assumed that phonon-frequency distribution is independent of polarization, for general polycrystalline media one can consider that $\rho(\omega)$ depends upon the direction of polarization and upon the kinds of atoms of the lattice. Then the inelastic-scattering cross section can be obtained by using our main formula repeatedly for various directions and averaging the obtained results. Currently, this has been attempted for graphite only where results depend only on the angle of the momentum-transfer vector with crystalline planes in graphite.(4) Averaging over this angle is done as the last step for the first two calculations and in the evaluation of multigroup scattering matrices averaging over this angle is done immediatelv orior to averagino over initial enfroies. 


\section{INITIAL CALCULATION OF SEVERAL PARAMETERS}

For simple cubic Bravais lattice the differential scattering cross section in the incoherent approximation can be written $(2,4)$ as

$$
d\left(E \rightarrow E^{\prime}, \theta\right)=\left(\sigma_{b} / 8 \pi^{2}\right)\left(E^{\prime} / E\right)^{1 / 2} \int_{-\infty}^{\infty} d t \exp \left\{-i t\left(E-E^{\prime}\right)+\mu_{\gamma}[g(t)-g(0)]\right\}
$$

where $E$ and $E^{\prime}$ are initial and final energy of the neutron; $\theta$ is the angle of scattering; $\sigma_{b}$ is the cross section for a bound atom; $\mu$ is the ratio of neutron mass and the mass of the atom; $\gamma$ is proportional to the square of momentum transfer:

$$
\gamma=E+E^{\prime}-2 \cos \theta \sqrt{E E^{\prime}}
$$

and $g(t)$ is a Fourier transform:

$$
g(t)=\int_{-\infty}^{\infty} \frac{d \omega}{\omega} \frac{\rho(\omega)}{\exp (\omega / k T)-1} e^{-i \omega t}
$$

Here $\rho(\omega)$ is assumed to be an even function of $\omega$. It is proportional to the number of modes of vibration of energy $\omega$, and it is normalized to unity, that is,

$$
\int_{0}^{\infty} \rho(\omega) d \omega=1
$$

Further, $k$ is the Boltzmann constant, $T$ the absolute temperature, and $[\exp (\omega / \mathrm{kT})-1]^{-1}$ is the average occupation number for a phonon of frequency $\omega$.

Operation with complex quantities in $g(t)$ can be avoided by shifting the path of integration. Substituting $T=t^{\prime}+(i / 2 k T)$, rearranging terms, and omitting primes, we can rewrite Eq. (1) as

$$
\sigma\left(E \rightarrow E^{\prime}, \theta\right)=\left(\sigma_{b} / 8 \pi^{2}\right)\left(E^{\prime} / E\right)^{1 / 2} \exp \left\{\left[\left(E-E^{\prime}\right) / 2 k T\right]-\mu \gamma g(0)\right\} \int_{-\infty}^{\infty} d t \exp \left\{-i t\left(E-E^{\prime}\right)+\mu \gamma\left(G^{\prime}(t)\right\},\right.
$$

where $G(t)$ is the even function defined by

$$
\begin{aligned}
& G(t)=\int_{0}^{\infty} f(\omega) \cos \omega t d \omega, \\
& g(0)=G(i / 2 k T)=\int_{0}^{\infty} f(\omega) \operatorname{ch}(\omega / 2 k T) d \omega,
\end{aligned}
$$


and

$$
f(\omega)=\frac{\rho(\omega)}{\omega s h(\omega / 2 k T)}
$$

In the present formulation of the problem, $\rho(\omega)$ will be given in unnormalized form at equidistant points:

$$
\rho_{u}(j \Delta \omega)=\rho_{j} \quad \text { for } \quad l \leq j \leq m-1 \quad .
$$

It will be assumed that

$$
\rho_{u}(0)=0 \text { and } \rho_{u}(j \Delta \omega)=0 \text { for } j \geq m
$$

Then we can compute

$$
f_{u}(j \Delta \omega)=f_{j}=\frac{\rho_{j}}{j \Delta \omega s h(j \Delta \omega / 2 k T)}, \text { for } \quad 1 \leq j \leq m-1,
$$

assuming $f_{0}$ as given. If $\rho$ is approximately parabolic for $\omega \leq \Delta \omega$,

$$
f_{0} \approx \rho_{1} \frac{2 k T}{(\Delta \omega)^{2}}
$$

If we assume now that $f_{u}$ has values given by Eq. (5) for integral multiples of $\Delta \omega$ and is linear in between, we can compute easily the normalization factor $N, g(0)$, and $G(t)$. With this assumption, $f_{u}$ is really a weighted sum of shifted rooflike functions:

$$
f_{u}=\sum f_{j} c^{(1)}(\omega-j \Delta \omega)
$$

where $c(1)(\omega)$ is a broken-line function equal to one for $\omega=0$ and vanishing for all other integral multiples of $\Delta \omega . G(t)$ is then

$$
\begin{aligned}
G(t) & =\int_{0}^{\infty} f(\omega) \cos \omega t d \omega \\
& =\frac{2 \Delta \omega}{N} \frac{1-\cos \Delta \omega t}{(\Delta \omega t)^{2}}\left[\frac{1}{2} f_{0}+\sum_{j=1}^{m-1} f_{j} \cos j \Delta \omega t\right],
\end{aligned}
$$

since

$$
\int_{-\infty}^{\infty} d \omega c^{(1)}(\omega) \cos \omega t=2 \int_{0}^{\Delta \omega} d \omega\left(1-\frac{\omega}{\Delta \omega}\right) \cos \omega t=2 \frac{1-\cos \Delta \omega t}{(\Delta \omega t)^{2}} .
$$


Replacing $t$ in Eq. (6) by $\mathrm{i} / 2 \mathrm{k} \mathrm{T}$, we obtain

$$
\begin{aligned}
g(0) & =\frac{1}{N} \int_{0}^{\infty} f_{u}(\omega) \operatorname{ch} \frac{\omega}{2 k T} d \omega \\
& =\frac{2 \Delta \omega}{N}\left(\frac{2 k T}{\Delta \omega}\right)^{2}\left(\operatorname{ch} \frac{\Delta \omega}{2 k T}-1\right)\left[\frac{1}{2} f_{0}+\sum_{j=1}^{m-1} f_{j} \operatorname{ch} j \frac{\Delta \omega}{2 k T}\right] .
\end{aligned}
$$

Then, differentiating both sides of Eq. (7) with respect to $1 / 2 \mathrm{kT}$, we obtain the normalization factor $\mathrm{N}$ :

$$
\begin{aligned}
\int_{0}^{\infty} f_{u}(\omega) \operatorname{sh} \frac{\omega}{2 k T} d= & \int_{0}^{\infty} \rho_{u}(a) d_{\mu}=N=2 \Delta u\left(\frac{2 k T}{\Delta \mu}\right)^{2}\left(\operatorname{ch} \frac{\Delta \omega}{2 k T}-1\right)\left[\sum_{j=1}^{m-1} \rho_{j}\right] \\
& +2(\Delta,)^{2}\left(\frac{2 k T}{\Delta \omega}\right)^{2}\left[\operatorname{sh} \frac{\Delta \omega}{2 k T}-2\left(\frac{2 k T}{\Delta \omega}\right)\left(\operatorname{ch} \frac{\Delta \omega}{2 k T}-1\right)\right] \\
& \times\left[\frac{1}{2} \hat{f}_{0}+\sum_{j=1}^{m-1} f_{j} \operatorname{ch~} \frac{\Delta \omega}{2 k T}\right]
\end{aligned}
$$

Thus, after having found $f_{j}$ from Eq. (5), $N$ from $\mathrm{Eq} \cdot(8)$, and $g(0)$ from Eq. (7), we are ready to "normalize" $f(\omega)$ :

$$
f_{j}^{(1)}=\frac{\Delta \omega}{2 N} f_{j}
$$

and to proceed with multiphonon expansion.

Since asymptotic expansion may be used in further calculations simultaneously with evaluation of Eqs. (5), (7), and (8), we compute also two other constants needed in Chapter $V$, Section $C$. These are the derivatives of $G(t)$ evaluated at $t=-i / 2 k T$. Taking Eq. (7) and differentiating it twice with respect to $1 / 2 k T$, we obtain 


$$
\begin{aligned}
a_{2}= & \int d \omega \rho(\omega)\left(\operatorname{coth} \frac{\omega}{2 k T}\right) \omega \\
= & \frac{2 \Delta \omega^{3}}{N}\left(\frac{2 k T}{\Delta \omega}\right)^{2}\left(\operatorname{ch} \frac{\Delta \omega}{2 k T}-1\right)\left[\sum_{j=1}^{m-1} j^{2} f_{j} \operatorname{chj} \frac{\Delta \omega}{2 k T}\right] \\
& +2 \frac{2 \Delta \omega^{2}}{N}\left(\frac{2 k T}{\Delta \omega}\right)^{2}\left[\operatorname{sh} \frac{\Delta \omega}{2 k T}-2\left(\frac{2 k T}{\Delta \omega}\right)\left(\operatorname{ch} \frac{\Delta \omega}{2 k T}-1\right)\right]\left[\sum_{j=1}^{m-1} \rho_{j}\right] \\
& +\frac{2 \Delta \omega^{3}}{N}\left(\frac{2 k T}{\Delta \omega}\right)^{2}\left[\operatorname{ch} \frac{\Delta \omega}{2 k T}-4\left(\frac{2 k T}{\Delta \omega}\right) \operatorname{sh} \frac{\Delta \omega}{2 k T}+6\left(\frac{2 k T}{\Delta \omega}\right)^{2}\left(\operatorname{ch} \frac{\Delta \omega}{2 k T}-1\right)\right] \\
& x\left[\frac{1}{2} f_{0}+\sum_{j=1}^{m-1} f_{j} \operatorname{chj} \frac{\Delta \omega}{2 k T}\right] .
\end{aligned}
$$

Differentiating once again, we have:

$$
\begin{aligned}
& a_{3}=\int d \omega \rho(\omega) \omega^{2} \\
& =\frac{2 \Delta \omega^{3}}{N}\left(\frac{2 k T}{\Delta \omega}\right)^{2}\left(\operatorname{ch} \frac{\Delta \omega}{2 k T}-1\right)\left[\sum_{j=1}^{m-1} j^{2} \rho_{j}\right] \\
& +3 \frac{2 \Delta \omega^{4}}{N}\left(\frac{2 k T}{\Delta \omega}\right)^{2}\left[\operatorname{sh} \frac{\Delta \omega}{2 k T}-2\left(\frac{2 k T}{\Delta \omega}\right)\left(\operatorname{ch} \frac{\Delta \omega}{2 k T}-1\right)\right]\left[\sum_{j=1}^{m-1} j^{2} f_{j} \operatorname{chj} \frac{\Delta \omega}{2 k T}\right] \\
& +3 \frac{2 \Delta \omega^{3}}{N}\left(\frac{2 k T}{\Delta \omega}\right)^{2}\left[\operatorname{ch} \frac{\Delta \omega}{2 k T}-4\left(\frac{2 k T}{\Delta \omega}\right) \operatorname{sh} \frac{\Delta \omega}{2 k T}+6\left(\frac{2 k T}{\Delta \omega}\right)^{2}\left(\operatorname{ch} \frac{\Delta \omega}{2 k T}-1\right)\right] \\
& x\left[\sum_{j=1}^{m-1} \rho_{j}\right]+\frac{2 \Delta \omega^{4}}{N}\left(\frac{2 k T}{\Delta \omega}\right)^{2}\left[\operatorname{sh} \frac{\Delta \omega}{2 k T}-6\left(\frac{2 k T}{\Delta \omega}\right) \operatorname{ch} \frac{\Delta \omega}{2 k T}+18\left(\frac{2 k T}{\Delta \omega}\right)^{2} \operatorname{sh} \frac{\Delta \omega}{2 k T}\right. \\
& \left.-24\left(\frac{2 k T}{\Delta \omega}\right)^{3}\left(\operatorname{ch} \frac{\Delta \omega}{2 k T}-1\right)\right]\left[\frac{1}{2} f_{0}+\sum_{j=1}^{m-1} f_{j} c h j \frac{\Delta \omega}{2 k T}\right] .
\end{aligned}
$$


In these expressions the first term is dominant. Evaluating other coefficients in front of $\Sigma$ symbols, we gain accuracy expressing the needed parameters in power series of the small constant $\Delta \omega / 2 \mathrm{kT}$.

At this stage we have computed $g(0), a_{2}, a_{3}, f_{j}^{(1)}$, and the scaling factor. For graphite $g(0), a_{2}, a_{3}$, and $f_{j}(1)$ are calculated separately for perpendicular vibrations using $\rho_{1}$, and for vibrations in the planes using $\rho_{2}$. Then, for every set of directions, $b$, the appropriate quantities are found by interpolating linearly with $\ell^{2}$ as described in Appendix C. Finally, for each $\ell$ the calculations proceed as is described in Chapters III, IV, and $\mathrm{V}$.

\section{THE MULTIPHONON EXPANSION}

The multiphonon expansion of Eq. (2) is obtained by expanding $\exp \mu \gamma \mathrm{G}(\mathrm{t})$ in a power series.

$$
\begin{aligned}
\sigma\left(E \rightarrow E^{\prime}, \theta\right)= & \left(\sigma_{b} / 8 \pi^{2}\right)\left(E^{\prime} / E\right)^{1 / 2} \exp \left\{\left[\left(E-E^{\prime}\right) / 2 k T\right]-\mu \gamma g(0)\right\} \\
& \int_{-\infty}^{\infty} \exp i\left(E-E^{\prime}\right) t d t \sum_{n=0}^{\infty} \frac{(\mu \gamma)^{n}}{n !}[G(t)]^{n}
\end{aligned}
$$

Using Eq. (6), with the understanding that $f_{-j}=f_{j}$, we can express

$$
[G(t)]^{n}=\left(\frac{2 \sin \Delta \omega t / 2}{\Delta \omega t}\right)^{2 n}\left[\sum_{j=-m+1}^{m-1} f_{j}^{(1)} \exp i j \Delta \omega t\right]^{n}
$$

as a product of two functions. The first function is independent of the specifications of the problem and has a Fourier transform which is an even function of the argument; this is nonvanishing only for argument values smaller than $n \Delta \omega$. In Appendix $A$ we have computed a table of transform values for integral multiples of $\Delta a$ :

$$
\frac{\Delta \omega}{2 \pi} \int_{-\infty}^{\infty}\left(\frac{2 \sin \Delta \omega t / 2}{\Delta \omega t}\right)^{2 n} \exp \left(-i \frac{\Delta \omega t}{2} 2 \nu\right) d t=c_{\nu}^{(n)}
$$

The second factor of Eq. (13) is a weighted sum of exponentials. By means of the abbreviation 


$$
\left[\sum_{j=-m+1}^{m-1} f_{j}^{(1)} \exp (i j \Delta \omega t)\right]^{n}=\sum_{j=-n(m-1)}^{n(m-1)} F_{j}^{(n)} \exp (i j \Delta \omega t),
$$

we find weighting factors $F_{j}^{(n)}$ by an iterative procedure:

$$
F_{j}^{(n)}=\sum_{i=-m+1}^{m-1} f_{i}^{(1)} F_{j-i}^{(n-1)} \text { for } 0 \leq j \leq n(m-1), \ldots
$$

where it is understood that

$$
F_{-j}^{(n)}=F_{j}^{(n)} \text { and } F_{j}^{(n)}=0 \text { for }|j|>n(m-1)
$$

Now substituting Eq. (15) into Eq. (13) and using Eq. (14), we obtain easily the Fourier transform of $[G(t)]^{n}$ :

$$
\frac{\Delta \omega}{\pi} \int_{0}^{\infty} d t \cos j \Delta u t[G(t)]^{n}=\sum_{\nu=-n+1}^{n-1} c_{\nu}^{(n)} F_{j-\nu}^{(n)}=f_{j}^{(n)}
$$

for $0 \leq \mathrm{j} \leq \mathrm{n} m-1$. Here again it will be understood that

$$
f_{-j}^{(n)}=f_{j}^{(n)} \text { and } f_{j}^{(n)}=0 \text { for }|j| \geq n m \text {. }
$$

Thus, the multiphonon contributions are determined using Eqs. (16) and (17).

This calculation of multiphonon contributions by means of Eqs. (16) and (17) is based on the assumption that $f$ can be represented as weighted sum of an elementary function displaced repeatedly by a constant interval. The coefficients $c_{\nu}^{(n)}$ have been evaluated by assuming that this elementary function is rooflike. If Dirac's $\delta$-function was chosen for the elementary function, the expressions for $g(0), N$, and $a_{2}$ would be much simplified, and Eq. (17) would be unnecessary. Only some simple modifications of present Eqs. (7), (8), (10), (11), and (14) would be needed if $f$ was approximated by a step function. 


\section{CALCULATIONS OF DIFEERENTIAL SCATTERING CROSS SECTION AND SCATTERING KERNEL}

If $\mathrm{E}$ and $\mathrm{E}^{\prime}$ are integral multiples of $\Delta \omega:$

$$
E=i \Delta \omega ; E^{\prime}=i^{\prime} \Delta l,
$$

the inelastic-scattering cross section may be obtained substituting Eq. (17) into Eq. (12):

$$
\sigma\left(E=1 \Delta \mu \rightarrow E^{\prime}=1^{\prime}\langle\alpha, \theta)=\left(\sigma_{b^{\prime}} 4 \pi \Delta \mu\right)\left(i^{\prime}, 1\right)^{2}{ }^{2}\left\{\exp \left[\left(1-1^{\prime}\right) \frac{\Delta u}{2 k T}-\mu \nu g(0)\right]\right\}\left[\sum_{n=1}^{\infty} \frac{1}{n !}\left(\mu \gamma^{n} f_{\left(1-1^{\prime}\right)}^{(n)}\right]\right.\right.
$$

The leading term in Eq. (12) for $\mathrm{n}=0$ is a Dirac 5 -function and represents purely elastic cross section:

$$
\sigma_{\mathrm{el}}\left(E=i \Delta \mu \rightarrow E^{\prime}=E, \theta\right)=\left(\sigma_{\mathrm{b}} / 4\right) \exp [-2 \mu g(0) L i(1-\cos \theta)] .
$$

Since contributions of only 25 phonons have been considered in evaluating the sum of $\mathrm{Eq}$. (18), we assume that remaining terms $\mathrm{a}_{26}, \mathrm{a}_{27}, \ldots$ decrease in geometric progression, and to the sum of 25 terms we add the value of estimated remainder:

$$
R=\frac{a_{25}^{2}}{a_{24}-a_{25}}
$$

if it is smaller than $10^{\%}$ of the sum. Otherwise, convergence is considered unsatisfactory. Actually, the remaining terms decrease somewhat faster than in geometric progression, and values obtained are slight overestimates.

Instead of the differential scattering cross section, we may evaluate the scattering kernel S. (7) This is a function of energy and momentum change, and is connected with the differential scattering cross section by

$$
\sigma\left(E \rightarrow E^{\prime}, \epsilon\right)=S\left(\sigma_{b} / 4 \pi\right)\left(E^{\prime} / E\right)^{1 / 2}(k T)^{-1} \cdot \exp \left[\left(E-E^{\prime}\right) / 2 k T\right] .
$$

Using Eq. (18) we see that

$$
S=\frac{k T}{\Delta \alpha} \exp [-\mu \gamma g(0)] \sum_{n=1}^{\infty} \frac{(\mu \gamma)^{n}}{n !} \frac{(n)}{i}\left(E-E^{\prime}\right) / \Delta a
$$

And it can be computed easily for any change of momentum and energy change in integer multiples of $\Delta \omega$. Egelstaff $(7)$ prefers to consider $S$ as a function of two dimensionless parameters: one proportional to the change of momentum, squared, 


$$
\alpha=\mu \% / k T
$$

another proportional to the change in energy:

$$
\beta=\left|E-E^{\prime}\right| / k T \text {. }
$$

Thus our calculation may be used to evaluate $S$ for any given $\alpha$ and for any sequence of values $\beta$, till Eq. (20) stops converging according to our criterion.

Quite often for evaluation of the cross section the Placzek(8) expansion is used. It consists of expanding exp $\{\mu \gamma[g(t)-g(0)]\}$ in power series of $\mu$ and performing the Fourier transform term by term. This expansion has been found very convenient for evaluation of the total cross section. We can understand that this should be so by keeping $\gamma$ constant and integrating over all real values of energy change $\epsilon$. Then

$$
\int d \epsilon \int(\exp i \epsilon t)\{\exp \mu \gamma[g(t)-g(0)]\} d t=2 \pi,
$$

and we need only the first term of power series in $\mu(n=0)$ to evaluate this integral. Similarly, if we again (incorrectly) let $\epsilon$ assume all positive and negative values, we need only $(n+1)$ terms to evaluate the $n$-th moment:

$$
\int \epsilon^{\mathrm{n}} \mathrm{d} \epsilon \int(\exp \mathrm{i} \in \mathrm{t})\{\exp [\mu \gamma(g(t)-g(0))]\} d t
$$

However, the Placzek expansion converges poorly for purely elastic cross sections:

$$
\int(\exp \mathrm{i} \epsilon t)\{\exp [-\mu \gamma g(0)]\} d t
$$

and therefore converges poorly for purely inelastic cross section. Indeed, if one uses only a number of terms of order $\mu \gamma g(0)$ (when it is large), either the elastic or total inelastic cross section becomes negative. Since here we are interested in the value of the cross section for a specified energy change, we have preferred multiphonon expansion with considerably better prospects for convergence as seen in the Appendix B.

\section{CALCULATION OF MULTIGROUP INELASTIC MATRICES AND TRANSPORT CROSS SECTIONS}

To obtain the multigroup inelastic matrices and transport cross sections, we perform the integration over direction of scattering, $\theta$, analytically. Either the multiphonon expansion is used if it converges satisfactorily, or an asymptotic expression is used. Integration over final energies and averaging over initial energies is performed numerically. 
A. Calculation by Multiphonon Expansion

Integrating Eq. (18) over the angle of scattering, we obtain

$$
\begin{aligned}
\sigma\left(i \Delta \omega \rightarrow \mathrm{i}^{\prime} \Delta \omega\right)= & {\left[\sigma_{\mathrm{b}} / 4 \mu \mathrm{g}(0)(\Delta \omega)^{2} \mathrm{i}^{\mathrm{i}}\right] \exp \left(\mathrm{i}-\mathrm{i}^{\prime}\right) \Delta \omega / 2 \mathrm{kT} \sum_{\mathrm{n}=1}^{\infty}[g(0)]^{-\mathrm{n}} \mathrm{f}_{\left(\mathrm{i}-\mathrm{i}^{\prime}\right)}^{(\mathrm{n})} } \\
& \left(\left\{\exp \left[-\mu \mathrm{g}(0) \Delta \omega\left(\sqrt{\mathrm{i}}-\sqrt{\mathrm{i}^{\prime}}\right)^{2}\right]\right\}\left\{1+\frac{1}{1 !} \mu \mathrm{g}(0) \Delta \omega\left(\sqrt{\mathrm{i}}-\sqrt{\mathrm{i}^{\prime}}\right)^{2}+\ldots+\frac{1}{\mathrm{n} !}\left[\mu \mathrm{g}(0) \Delta \omega\left(\sqrt{\mathrm{i}}-\sqrt{\mathrm{i}^{\prime}}\right)^{2}\right]^{\mathrm{n}}\right\}\right. \\
& \left.-\left\{\exp \left[-\mu \mathrm{g}(0) \Delta \omega\left(\sqrt{\mathrm{i}}+\sqrt{\mathrm{i}^{\prime}}\right)^{2}\right]\right\}\left\{1+\frac{1}{1 !} \mu \mathrm{g}(0) \Delta \omega\left(\sqrt{\mathrm{i}}-\sqrt{\mathrm{i}^{\prime}}\right)^{2}+\ldots+\frac{1}{\mathrm{n} !}\left[\mu \mathrm{g}(0) \Delta \omega\left(\sqrt{\mathrm{i}}+\sqrt{\mathrm{i}^{1}}\right)^{2}\right]^{\mathrm{n}}\right\}\right) .
\end{aligned}
$$

Similarly, integration over $\theta$ of the purely elastic cross section, Eq. (19), gives

$$
\sigma_{e l}(i \Delta \omega)=\left[\sigma_{b} / 4 \mu g(0) \Delta \omega i\right]\{1-\exp [-4 \mu g(0) \Delta \omega i]\} \quad .
$$

We evaluate Eq. (21) using 25 terms and estimate the remainder by means of the assumption that neglected terms decrease in geometric progression as in Chapter IV. If the remainder turns out to large, we switch to the asymptotic formula of section B below. As seen in Appendix B, the multiphonon expansion is expected to be good even at very high energies if the energy change is not large and a sufficient number of phonons has been used; $(25=) n_{\max } \geq 4 a_{2} g(0)$.

In this part we evaluate also the transport cross section. We define the contribution of inelastic scattering to the transport cross section as

$$
\sigma_{t r}^{\prime \prime}(E)=\int d E^{\prime} \sigma_{t r}\left(E \rightarrow E^{\prime}\right)=\int d E^{\prime} \int \sigma\left(E \rightarrow E^{\prime}, \theta\right)(1-\cos \theta) 2 \pi d \cos \theta .
$$

And we obtain $\sigma_{\operatorname{tr}}\left(E \rightarrow E^{\prime}\right)$ using Eq. (18):

$$
\begin{aligned}
& \sigma_{t r}\left(E \rightarrow E^{\prime}\right)=\left[\sigma_{b} / 8 \mu^{2} g(0)^{2} \Delta \omega^{3} i^{3 / 2} i^{1}{ }^{1 / 2}\right] \exp \left[\left(i-i^{\prime}\right) \Delta \omega / 2 k T\right] \\
& \sum_{n=1}^{\infty}\left\{n[g(0)]^{-n+1} f_{\left(i-i^{\prime}\right)}^{(n-1)}-\mu g(0) \Delta \omega\left(\sqrt{i}-\sqrt{i^{\prime}}\right)^{2} \cdot[g(0)]^{-n} f_{\left(i-1^{\prime}\right)}^{(n)}\right\} \\
& \left(\left\{\exp \left[-\mu g(0) \Delta \omega\left(\sqrt{\mathrm{i}}-\sqrt{\mathrm{i}^{\prime}}\right)^{2}\right]\right\}\left\{1+\frac{1}{1 !} \mu \mathrm{g}(0) \Delta \omega\left(\sqrt{\mathrm{i}}-\sqrt{\mathrm{i}^{1}}\right)^{2}+\ldots+\frac{1}{\mathrm{n} !}\left[\mu \mathrm{g}(0) \Delta \omega\left(\sqrt{\mathrm{i}}-\sqrt{\mathrm{i}^{\prime}}\right)^{2}\right]^{\mathrm{n}}\right\}\right. \\
& \left.-\left\{\exp \left[-\mu g(0) \Delta \omega\left(\sqrt{\mathrm{i}}+\sqrt{\mathrm{i}^{\prime}}\right)^{2}\right]\right\}\left\{1+\frac{1}{1 !} \mu g(0) \Delta \omega\left(\sqrt{\mathrm{i}}+\sqrt{\mathrm{i}^{\prime}}\right)^{2}+\ldots+\frac{1}{\mathrm{n} !}\left[\mu \mathrm{g}(0) \Delta \omega\left(\sqrt{\mathrm{i}}+\sqrt{\mathrm{i}^{\prime}}\right)^{2}\right]^{\mathrm{n}}\right\}\right) \text {, }
\end{aligned}
$$


where it is understood that $f_{\left(i-i^{\prime}\right)}^{(0)}=0$. Since evaluation of Eq. (23) may be performed at the same time as evaluation of $\mathrm{Eq}$. (21), not much additional computation is required. Also, the computation may be arranged so that evaluation of the long sum is done only for $i>i^{\prime}$ and the results used for upscattering, $i^{\prime}>i$. Later integration over final energies of the transport cross section is described. To this sum we add also the contribution of purely elastic scattering:

$$
\sigma_{t r, e l}=\left[\sigma_{b} / 8(\mu g(0) \Delta \omega i)^{2}\right] \cdot\{1-[1+4 \mu g(0) \Delta \omega i] \exp [-4 \mu g(0) \Delta \omega i]\}
$$

B. Calculation by an Asymptotic Expression

When energy change and initial energy are large, the multiphonon expansion fails to converge, and we use an asymptotic expression to calculate $\sigma\left(E \rightarrow E^{\prime}\right)$. The asymptotic expression can be obtained in a closed form by integrating $\mathrm{Eq}$. (2) over the directions of scattering:

$$
\begin{aligned}
\sigma\left(E \rightarrow E^{\prime}\right)= & \left(\sigma_{b} / 8 \pi \mu E\right) \quad \int_{-\infty}^{+\infty}\left\{\exp \left[-i\left(E-E^{\prime}\right)\left(t+\frac{i}{2 k T}\right)\right]\right\} \\
& \frac{\exp \left\{\mu\left(\sqrt{E}+\sqrt{E^{\prime}}\right)^{2}[G(t)-g(0)]\right\}-\exp \left\{\mu\left(\sqrt{E}-\sqrt{E^{\prime}}\right)^{2}[G(t)-g(0)]\right\}}{G(t)-g(0)} d t .
\end{aligned}
$$

We know that for very large energies the cross section approaches the cross section of a free atom. The downscattering cross section is appreciable only when $E-E^{\prime} \lesssim\left(\sqrt{E}+\sqrt{E^{\prime}}\right)^{2}$ and very nearly vanishes for larger energy losses. Thus, it seems that the behavior of the cross section in the drop-off region is most important when energies are not so very high. In this region the integral of the first term is very much larger than the integral of the second term $(\mu<1)$, as one can see clearly by trying to apply the method of steepest descent. To obtain the first term we expand $G(t)$ in Taylor series about the point $t=-i / 2 k T$ :

$G(t)=g(0)+i\left(t+\frac{i}{2 k T}\right)-\frac{1}{2 !} a_{2}\left(t+\frac{i}{2 k T}\right)^{2}-\frac{i}{3 !} a_{3}\left(t+\frac{i}{2 k T}\right)^{3}+\frac{1}{4 !} a_{4}\left(t+\frac{i}{2 k T}\right)^{4}+\ldots$

where

$$
\begin{aligned}
g(0) & =\int \operatorname{d} \omega \rho(\omega)\left(\operatorname{coth} \frac{\omega}{2 k T}\right) \frac{1}{\omega} & a_{2} & =\int \operatorname{d\omega } \rho(\omega)\left(\operatorname{coth} \frac{\omega}{2 k T}\right) \omega \\
1 & =\int \operatorname{d\omega } \rho(\omega) & a_{3} & =\int \operatorname{d\omega } \omega(\omega) \omega^{2}
\end{aligned}
$$

are constants evaluated in Chapter II. 
Now, if we substitute Eq. (27) into the integrand and introduce a new variable of integration,

$$
x=\sqrt{\frac{1}{2} \mu\left(\sqrt{E}+\sqrt{E^{\prime}}\right)^{2} a_{2}}\left(t+\frac{i}{2 k T}\right),
$$

we see that the integral in Eq. (26) is very nearly equal to

$$
\begin{aligned}
& \int d t \frac{\exp \left\{-i\left(E-E^{\prime}\right)\left(t+\frac{i}{2 k T}\right)+\mu(\sqrt{E}+\sqrt{E})^{2}[G(t)-g(0)]\right\}}{G(t)-g(0)}= \\
& \int\left\{\text { is }-\sqrt{\frac{a_{2}}{2 \mu\left(\sqrt{E}+\sqrt{E^{\prime}}\right)^{2}}} x^{2}-\frac{a_{2}}{2_{2}\left(\sqrt{E}+\sqrt{E^{\prime}}\right)^{2}} \frac{2}{3} i \frac{a_{3}}{a_{2}^{2}} x^{3}+\ldots\right\}^{-1} \\
& \exp \left\{-2 \frac{\sqrt{E}-\sqrt{E^{\prime}}-\mu\left(\sqrt{E}+\sqrt{E^{\prime}}\right)}{\sqrt{2_{1, a_{2}}}} i x-x^{2}-\sqrt{\frac{a_{2}}{2\left(\sqrt{E}+\sqrt{E^{\prime}}\right)^{2}}} \frac{2}{3} i \frac{a_{3}}{a_{2}^{2}} x^{3}\right. \\
& \left.+\frac{a_{2}}{2 \mu\left(\sqrt{E}+\sqrt{E^{\prime}}\right)^{2}} \frac{1}{3} \frac{a_{4}}{a_{2}^{3}} x^{4}+\ldots\right\} d x
\end{aligned}
$$

If we assume now that

$$
\frac{\sqrt{E}-\sqrt{E^{\prime}}-\mu\left(\sqrt{E}+\sqrt{E^{\prime}}\right)}{\sqrt{2 \mu a_{2}}}=\eta
$$

is finite, while $2 \mu\left(\sqrt{E}+\sqrt{E^{\prime}}\right)^{2} / a_{2} \rightarrow \infty$, and expand the integrand in power series, we find that leading term reduces to a standard form. (9) The value of the integral can be obtained easily from the integral

$$
\int_{-\infty}^{\infty} d x \exp \left[-2 i \eta x-x^{2}\right]=\sqrt{\pi} \exp \left(-\eta^{2}\right)
$$

by integration with respect to the parameter $\eta$. The constant of integration is determined from consideration of the value of the integral for large positive $\eta$. Then the method of steepest descent shows that the integral vanishes when the path of integration is below the pole. We obtain, thus,

$$
\int \frac{d x}{i x} \exp \left[-2 i \eta x-x^{2}\right]=\pi(1-\operatorname{erf} \eta)
$$

Integration of succeeding terms is elementary. Collecting the terms, we obtain for $E>E^{\prime}$, 


$$
\begin{aligned}
\sigma\left(E \rightarrow E^{\prime}\right)= & \left(\sigma_{\mathrm{b}} / \beta_{\mu} \mathrm{E}\right)\left\{1-\operatorname{erf} r_{1}-\sqrt{\frac{1}{\pi}} \sqrt{\frac{a_{2}}{2 \mu\left(\sqrt{E}+\sqrt{E^{\prime}}\right)^{2}}} e^{-\eta^{2}} \cdot\left[1+\frac{a_{3}}{3 a_{2}^{2}}-\frac{2 a_{3}}{3 a_{2}^{2}} \cdots^{2}\right]\right. \\
& +\sqrt{\frac{1}{\pi}}\left(\frac{a_{2}}{2 \mu\left(\sqrt{E}+\sqrt{E^{\prime}}\right)^{2}}\right) e^{-\eta^{2}}\left[\left(1-\frac{2}{3} \frac{a_{3}}{a_{2}^{2}}-\frac{1}{2} \frac{a_{4}}{a_{2}^{3}}+\frac{5}{6} \frac{a_{3}^{2}}{a_{2}^{4}}\right) \eta\right. \\
& \left.\left.+\left(\frac{1}{3} \frac{a_{4}}{a_{2}^{3}}-\frac{10}{9} \frac{a_{3}^{2}}{a_{2}^{4}}\right) \eta^{3}+\frac{2}{9} \frac{a_{3}^{2}}{a_{2}^{\frac{4}{2}}} \eta^{5}\right]+\ldots\right\} .
\end{aligned}
$$

Equation (30) is considered unsatisfactory and not usable when $\eta$ becomes so large and positive that the second term is larger in absolute value than the first. Neglected values are considered vanishingly small. In practice, we have neglected the last term for simplicity, and we have used Eq. (30) only for downscattering. Upscattering has been obtained from Eq. (30) by means of detailed balance:

$$
\sigma\left(E^{\prime} \rightarrow E\right)=\sigma\left(E \rightarrow E^{\prime}\right) \frac{E}{E^{\prime}} \exp \left[-\left(E-E^{\prime}\right) / k T\right]
$$

For large energies $\sigma_{\operatorname{tr}}\left(E \rightarrow E^{\prime}\right)$ can be calculated in a very similar way. Direct integration using $\mathrm{Eq}$. (2) for $\sigma\left(\mathrm{E} \rightarrow \mathrm{E}^{\prime}, \theta\right)$ gives

$$
\begin{aligned}
\sigma_{t r}\left(E \rightarrow E^{\prime}\right)= & \int \sigma\left(E \rightarrow E^{\prime}, \theta\right)(1-\cos \theta) 2 \pi d \cos \theta=\left(\sigma_{b} / 8 \pi i \omega E\right) \int \frac{d t \exp \left[-i\left(E-E^{\prime}\right)\left(t+\frac{i}{2 k T}\right)\right]}{G(t)-g(0)} \\
& \cdot\left\{\left[2-\frac{1}{2 \mu \sqrt{E E^{\prime}}[G(t)-g(0)]}\right] \exp \left[\mu\left(\sqrt{E}+\sqrt{E^{\prime}}\right)^{2}(G(t)-g(0))\right]\right. \\
& \left.-\frac{1}{2 \mu \sqrt{E E^{\prime}}[G(t)-g(0)]} \exp \left[\mu\left(\sqrt{E}-\sqrt{E^{\prime}}\right)^{2}(G(t)-g(0))\right]\right\} .
\end{aligned}
$$

Here again the integral of the second term is very small, and we can evaluate the first term by the same procedure as previously. An additional singular integral is encountered and is evaluated by integrating Eq. (29) with respect to the parameter $\eta$ :

$$
\int \frac{d x}{x^{2}} \exp \left(-2 i \eta x-x^{2}\right)=2 \pi\left\{\eta[1-\operatorname{erf} \eta]-\frac{1}{\sqrt{\pi}} \exp \left(-\eta^{2}\right)\right\} .
$$

The result of this integration is a sum of two series. The first one is just twice the series of Eq. (30), representing predominantly backward scattering for $E-E^{\prime} \geq \mu\left(\sqrt{E}+\sqrt{E^{\prime}}\right)^{2}$. The second series represents the deviation 
from backward scattering and tends to cancel the value of the first series when $E^{\prime} \rightarrow E$ and scattering becomes nearly forward. Thus, simultaneously with Eq. (30), we may evaluate also

$$
\begin{aligned}
\sigma_{\operatorname{tr}}\left(E \rightarrow E^{\prime}\right)= & \left(c_{b}, 4 \mu E\right)\left(1-\operatorname{erf} ;-\sqrt{\frac{a_{2}}{2 \pi \mu\left(\sqrt{E}+\sqrt{E^{1}}\right)^{2}}} e^{-\eta^{2}}\left[1+\frac{a_{3}}{3 a_{2}^{2}}-\frac{2 a_{3}}{3 a_{2}^{2}} \eta^{2}\right]+\ldots\right. \\
& -\sqrt{\frac{\left(\sqrt{E}+\sqrt{\left.E^{1}\right)^{2} a_{2}}\right.}{8 \mu E E^{\prime}}\left\{\eta(\operatorname{erf} \eta-1)+\frac{1}{\sqrt{\pi}} e^{-\eta^{2}}+\sqrt{\frac{a_{2}}{2 \pi \mu\left(\sqrt{E}+\sqrt{E^{1}}\right)^{2}}}\right.} \\
& {\left.\left.\left[\sqrt{\pi}(\operatorname{erf} \eta-1)+\frac{a_{3}}{3 a_{2}^{2}} \eta e^{-\eta^{2}}\right]+\ldots\right\}\right) }
\end{aligned}
$$

\section{Integration over Final Energies and Averaging over Initial Energies}

To develop multigroup scattering cross sections, we numerically integrate over final energies $E^{\prime}$ and average over initial energies $E$ by means of Simpson's rule. Thus, in every energy group there has to be an even number of elementary intervals. At first, integrations over $E^{\prime}$ are performed for every value of $E$. The results of these integrations, for every value of $\mathrm{E}$, are inelastic cross sections for every energy group and scattering contribution to the transport cross section $\sigma t_{r}$. To obtain the latter, we integrate over $\mathrm{E}^{\prime}$ of Eqs. (23) or (31) and add the elastic contribution Eq. (24). To economize the calculations, for every pair of values, $E$ and $E^{\prime}$, the evaluation of inelastic cross section and transport cross section for up-and down-scattering is done at the same time, and the results are multiplied with appropriate coefficients and accumulated. Integration begins with $E=E^{\prime}=\Delta \omega$. Then $E$ is kept the same and $E^{\prime}$ increases till maximum value is reached or the asymptotic formula Eq. (30) fails and the cross section is considered negligible for larger values of $\mathrm{E}^{\prime}$. At the end of this step, we have a complete set of cross sections for $E=\Delta \omega$. In the next step, we start with $E=E^{\prime}=2 \Delta \omega$ and end up with a complete set of cross sections for $E=2 \Delta \alpha$. We continue in this way, always starting evaluation on the diagonal, till the maximum value of $E$ is reached.

After finishing integration over $E^{\prime}$, with the first value of $l$, we pick up the next value of $l$, as explained in Appendix $C$. Interpolation takes place for new values of contstants $f_{j}^{(3)}, g(0), a_{2}$, and $a_{3}$; we repeat the calculations of Chapter $\mathrm{V}$ sections $\mathrm{A}$ and $\mathrm{B}$, and integrate over final energy $\mathrm{E}^{\prime}$. The results of this integration are immediately multiplied with appropriate weighting factor for each $\ell$ and immediately added to the previous values.

Final results may be used to obtain standard multigroup crosssection sets for reactor regions having various flux shapes. In this, last, part of the procedure, the complete transport cross section: 


$$
\sigma_{t r}=\sigma_{t r}^{\prime}+\sigma_{c}
$$

is calculated. The capture cross section is assumed to be proportional to $\mathrm{E}^{-1 / 2}$, and its value for $2,200 \mathrm{~m} / \mathrm{sec}$ neutrons is assumed as given. Then $\sigma_{C}$, the diffusion coefficient $\left(1,3 \sigma_{t r}\right)$, and the inelastic scattering cross sections for every group of final energy $E^{\prime}$ are averaged in every group of initial energy $E$, weighting each with a chosen flux. So far three forms of the flux have been chosen in each group:

1. Hardened Maxwellian:

$\phi \propto(\mathrm{CE} / \mathrm{kT}) \exp (-\mathrm{CE} / \mathrm{kT})$

where $C$ is a number somewhat larger than one. This form is convenient for groups of lower energy.

2. The flux is assumed to be proportional to the $\mathrm{N}$-th power of energy:

$$
\phi \propto(E / k T)^{N}
$$

3. The flux is given numerically for every value of energy within the group, for which cross sections are calculated.

Calculation of the cross sections for every couple of $E$ and $E^{\prime}$ that can be expressed in integral multiples of $\Delta \omega$ may be too time consuming and, indeed, unnecessary if energies are large. From the leading term in Eq. (30) we see that the extent of the drop-off region at large energies is proportional to the square root of the initial energy. Thus, at high energies, the elementary interval of integration may be allowed to increase proportionally to the square root of energy. The increase, however, must be such that the number of elementary integration intervals in every group is even. 
Appendix A

EVALUATION OF $c_{\nu}^{(n)}$

After a change of integration variable Eq. (14) can be written as

$$
c_{\nu}^{(n)}=\frac{1}{\pi} \int_{-\infty}^{+\infty}\left(\frac{\sin x}{x}\right)^{2 n} \exp (-2 i \nu x) d x
$$

This integral can be evaluated exactly by changing slightly the path of integration to avoid $x=0$, expanding $(\sin x)^{2 n}$ in power series of exp ix and finding the residue of each term. In this way we obtain $(10)$

$$
\begin{aligned}
c_{\nu}^{(n)}= & \frac{1}{(2 n-1) !} \\
& {\left[(n-\nu)^{2 n-1}-\left(\begin{array}{c}
2 n \\
1
\end{array}\right)(n-\nu-1)^{2 n-1}+\left(\begin{array}{c}
2 n \\
2
\end{array}\right)(n-\nu-2)^{2 n-1}-\left(\begin{array}{c}
2 n \\
3
\end{array}\right)(n-\nu-3)^{2 n-1}+\cdots+(-1)^{n-\nu-1}\left(\begin{array}{c}
2 n \\
n-\nu-1
\end{array}\right)(1)^{2 n-1}\right] . }
\end{aligned}
$$

We see also while deriving this formula that $c_{\nu}^{(n)}=0$ for $|\nu| \geq n$. Further, we can show simply, starting with Eq. (A-1) and summing over all integer values of $\nu$, that

$$
c_{0}^{(n)}+2 \sum_{\nu=1}^{n-1} c_{\nu}^{(n)}=1
$$

Table A-1 contains values of $c_{\nu}^{(n)}$ derived by direct evaluation of Eq. (A-2). All values contained therein satisfy Eq. (A-3) coincident with 8-place accuracy. For $1 \leq n \leq 11$, values of $(2 n-1) ! c_{\nu}^{(n)}$ were found exactly. By this time, however, the calculations were involving numbers as high in order of magnitude as $10^{22}$. The ensuing calcula-

\begin{tabular}{|r|c|c|}
\hline$n$ & $v$ & Digits Lost \\
\hline 5 & 0 & 1 \\
10 & 0 & 3 \\
15 & 0 & 4 \\
20 & 0 & 6 \\
25 & 0 & 8 \\
25 & 5 & 4 \\
25 & 10 & 1 \\
25 & 15 & 1 \\
25 & 20 & 0 \\
\hline
\end{tabular}
tions $(12 \leq \mathrm{n} \leq 25)$ were continued with the intention of guaranteeing only 8-place accuracy.

Since the series in $\mathrm{Eq} .(\mathrm{A}-2)$ is alternating in sign and since the binomial coefficients increase with successive terms, there was a tendency toward cancellation dependent upon the values of $\mathrm{n}$ and $\nu$. For a given value of $\mathrm{n}$ this tendency reduced with increasing values of $\nu$. It increased, however, with increasing values of $\mathrm{n}$. The adjoining tabulation is intended to exemplify this effect. The third column designates the number of digits lost from the largest term in the respective series. 
Table A-1

COEFFICIENTS OF $\varepsilon_{2}^{(n)}$

\begin{tabular}{|c|c|c|c|c|c|c|c|c|c|c|c|c|c|c|c|c|c|c|c|c|c|c|c|}
\hline$n$ & $v$ & $c_{2}^{\ln } \times 109$ & G & $n$ & $v$ & $c_{v}^{\ln } \times 10^{n}$ & 9 & $n$ & $z$ & $c_{2}^{(n)} \times 100$ & 9 & $n$ & $y$ & $c_{\nu}^{(m)} \times 30^{9}$ & 9 & $n$ & $\nu$ & $c_{2}^{\ln x} \times 10^{4}$ & $q$ & $n$ & $y$ & $c_{y}^{i n !} \times 10^{4}$ & 9 \\
\hline \multirow[t]{2}{*}{1} & 0 & 10000000 & 0 & 10 & 4 & 2.2018669 & 3 & 14 & 12 & 1.2326137 & 20 & 18 & 4 & 1.6016700 & 2 & 21 & 2 & 1.2078808 & I & 23 & 15 & 3.2386105 & 16 \\
\hline & & & & & 5 & 1.1341319 & 4 & & 13 & 9.1836902 & 29 & & 5 & $3.456 \quad 1876$ & 3 & & 3 & 5.9365216 & 2 & & 16 & 85481443 & 19 \\
\hline \multirow[t]{3}{*}{2} & 0 & 6.6606667 & 1 & & 6 & 2.0693903 & 6 & & & & & & 6 & 5.1054171 & 4 & & 4 & $2.178 \quad 0373$ & 2 & & 17 & 8.5803055 & 22 \\
\hline & 1 & 1.6606667 & $i$ & & 7 & $9.468 \quad 3295$ & 9 & 15 & 0 & $2.510 \quad 4851$ & 1 & & 7 & 5.0252908 & 5 & & 5 & $5.913 \quad 5241$ & 3 & & 18 & $2.371 \quad 1955$ & 25 \\
\hline & & & & & 8 & 4.3098160 & 12 & & 1 & 2.0630735 & 1 & & 8 & 3.1778930 & 6 & & 6 & 1.1742268 & 3 & & 19 & 1.0347610 & 29 \\
\hline \multirow[t]{4}{*}{3} & 0 & 5.5000000 & 1 & & 9 & 8.2206352 & 18 & & 2 & 1.1414048 & 1 & & 9 & 1.2284490 & 7 & & 7 & 1.6786922 & 4 & & 20 & 2.4697009 & 35 \\
\hline & 1 & $2.166 \quad 6667$ & 1 & & & & & & 3 & $4.210 \quad 9370$ & 2 & & 10 & 2.7087243 & 9 & & 8 & 1.6931210 & 5 & & 21 & 2.9412906 & 43 \\
\hline & 2 & $8.333 \quad 3333$ & 3 & 11 & 0 & 2.9262269 & 1 & & 4 & 1.0185923 & 2 & & 11 & 3.0848030 & 11 & & 9 & 1.1734829 & 6 & & 22 & 8.3596509 & 57 \\
\hline & & & & & 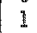 & 2.2428008 & 1 & & 5 & 1.5743122 & 3 & & 12 & 1.5629703 & 13 & & 10 & 5.4008861 & 8 & & & & \\
\hline \multirow[t]{5}{*}{4} & 0 & 4.7936507 & 1 & & 2 & 1.0019429 & $i$ & & 6 & 1.4974105 & 4 & & 13 & 27754497 & 16 & & II & 1.5777967 & 9 & 24 & 0 & 1.9884680 & 1 \\
\hline & 1 & 2.3630952 & 1 & & 3 & 2.5451983 & 2 & & 7 & $8.304 \quad 3229$ & 6 & & 14 & 1.1407854 & 19 & & 12 & 2.7535017 & 11 & & I & 1.7574395 & 1 \\
\hline & 2 & 2.3809523 & 2 & & 4 & $3.511 \quad 1077$ & 3 & & 8 & 2.4818502 & 7 & & 15 & 4.8417295 & 24 & & 13 & 2.6397162 & 13 & & 2 & 1.2123671 & i \\
\hline & 3 & 1.9841269 & 4 & & 5 & $2.436 \quad 1241$ & 4 & & 9 & 3.5493851 & 9 & & 16 & $\begin{array}{lll}3.325 & 1955\end{array}$ & 30 & & 14 & 1.2327358 & 15 & & 3 & 6.5128957 & 2 \\
\hline & & & & & 6 & $7.496 \quad 5178$ & 6 & & 10 & 2.0091861 & 11 & & 17 & 9.6775929 & 41 & & 15 & 2.3406061 & 18 & & 4 & 2.7138567 & 2 \\
\hline \multirow[t]{6}{*}{5} & 0 & 4.3041776 & 1 & & 7 & 8.1587910 & 8 & & 11 & 3.2365913 & 14 & & & & & & 16 & 1.3533108 & 21 & & 5 & 8.7215228 & 3 \\
\hline & 1 & 2.4314925 & 1 & & 8 & 2.0383683 & 10 & & 12 & 7.7602485 & 18 & 19 & 0 & 2.2329949 & 1 & & 17 & 1.4450840 & 25 & & 6 & $2.145 \quad 1351$ & 3 \\
\hline & 2 & 4.0255731 & 2 & & 9 & 4.1047002 & 14 & & 13 & 6.0719897 & 23 & & 1 & 1.9113283 & 1 & & 18 & 1.0902884 & 30 & & 7 & 3.9981124 & 4 \\
\hline & 3 & 1.3833774 & 3 & & 10 & 1.9572941 & 20 & & 14 & 1.1309962 & 31 & & 2 & 1.1668001 & $\overline{1}$ & & 19 & 6.5735640 & 38 & & 8 & 5.57590006 & 5 \\
\hline & 4 & 2.7557319 & 6 & & & & & & & & & & 3 & $5.456 \quad 4285$ & 2 & & 20 & 2,9893107 & 50 & & 9 & $5.727 \quad 3232$ & 6 \\
\hline & & & & 12 & 0 & $2.803 \quad 2619$ & 1 & 16 & 0 & 2.4315338 & 1 & & 4 & 1.7967629 & 2 & & & & & & 10 & 4.2475324 & 7 \\
\hline \multirow[t]{7}{*}{6} & 0 & 3.9392556 & 1 & & 1 & 2.1956830 & 1 & & 1 & 2.0224454 & 1 & & 5 & 4.2226622 & 3 & 22 & 0 & $2.076 \quad 2933$ & 1 & & 11 & $2.218 \quad 792$ & 8 \\
\hline & 1 & 2.4396028 & 1 & & 2 & 1.0487418 & I & & 2 & 1.1607955 & 1 & & 6 & 6.9665316 & 4 & & 1 & 1.8148278 & I & & 12 & 7.9035283 & 10 \\
\hline & 2 & 5.5202020 & 2 & & 3 & $2,999^{\circ} 4159$ & 2 & & 3 & $4.561 \leadsto 397$ & 2 & & 7 & $7.89 \mathrm{l} \quad 4769$ & 5 & & 2 & 1.2107259 & 1 & & 13 & 1.8443836 & 11 \\
\hline & 3 & 3.8238786 & 3 & & 4 & 4.9523095 & 3 & & $a$ & 1.2104051 & 2 & & 8 & 5.9587571 & 6 & & 3 & $6.1462 \% 8$ & 2 & & 14 & 2.6726183 & 13 \\
\hline & 4 & 5.1006092 & 5 & & 5 & $4.473 \quad 1411$ & 4 & & 5 & $2.124 \quad 1798$ & 3 & & 9 & $2.883 \quad 1681$ & 7 & & 4 & 2.3621280 & 2 & & 15 & 2.2388184 & 15 \\
\hline & 5 & 2.5052108 & 8 & & 6 & 2.0225084 & 5 & & 6 & 2.3929595 & 4 & & 10 & 8.4740266 & 9 & & 5 & 6.8211776 & 3 & & 16 & 9.8216928 & 18 \\
\hline & & & & & 7 & 3.9679867 & 7 & & 7 & 1.6594236 & 5 & & 11 & 1.4049088 & 10 & & 6 & 1.4650822 & 3 & & 17 & 1.9582622 & 20 \\
\hline \multirow[t]{8}{*}{7} & 0 & 3.6537086 & 1 & & 8 & 2.6346734 & 9 & & 8 & $6.676 \quad 1530$ & 7 & & 12 & 1.1814668 & 12 & & 7 & 2.3095246 & 4 & & 18 & 1.4337300 & 23 \\
\hline & 1 & 2.4178841 & 1 & & 9 & 3.6338306 & 12 & & 0 & $1.430 \quad 3578$ & 8 & & 13 & $4.296 \quad 4269$ & 15 & & 8 & 2.6265640 & 5 & & 19 & 2.7437287 & 27 \\
\hline & 2 & $6.797 \quad 4968$ & 2 & & 10 & 3.2448470 & 16 & & 10 & 1.4346824 & 10 & & 14 & $5.234 \quad 1896$ & 18 & & 9 & 2.1082343 & 6 & & 20 & $7.658 \quad 1532$ & 32 \\
\hline & 3 & $7.312 \quad 2366$ & 3 & & 11 & 3.8681701 & 23 & & 11 & 5.4839277 & 13 & & 15 & $\begin{array}{lll}1.371 & 1634\end{array}$ & 21 & & 10 & 1.1611275 & 7 & & 21 & 1.0280904 & 37 \\
\hline & 4 & 2.3762984 & 4 & & & & & & 12 & 5.5843506 & 16 & & 16 & 3.2714818 & 26 & & 11 & $4.230 \% 008$ & 9 & & 22 & 5.4417959 & 46 \\
\hline & 5 & 1.3133086 & 6 & 13 & 0 & 2.6945977 & 1 & & 13 & 7.5108451 & 20 & & 17 & 9.9855722 & 33 & & 12 & 9.7229842 & 11 & & 23 & 38466285 & 60 \\
\hline & 6 & 1.6059043 & 10 & & 1 & 2.1498081 & 1 & & 14 & 2.6116085 & 25 & & 18 & $7.265 \quad 4602$ & 44 & & 13 & $1.321 \quad 4473$ & 12 & & & & \\
\hline & & & & & 2 & 1.0865617 & $i$ & & 15 & $1.216 \quad 1250$ & 34 & & & & & & 14 & 9.7192876 & 15 & 25 & 0 & 1.9485379 & 1 \\
\hline 8 & 0 & 3.4224025 & 1 & & 3 & 3.4279354 & 2 & & & & & 20 & 0 & 2.1768871 & 1 & & 15 & 3.4061660 & 17 & & 1 & 1.7305825 & 1 \\
\hline & 1 & 2.3812319 & 1 & & 4 & 6.5730455 & 3 & 17 & 0 & 2.3595908 & 1 & & 1 & 1.8776616 & 1 & & 26 & $4.69654 A 6$ & 20 & & 2 & 1.2115719 & I \\
\hline & 2 & 7.8595252 & 2 & & 5 & 7.3499505 & 4 & & 1 & 1.9836513 & $i$ & & 2 & 1.2033457 & 1 & & 17 & $1.876 \quad 1225$ & 23 & & 3 & 6.6725185 & 2 \\
\hline & 3 & 1.1502774 & 2 & & 6 & 4.4859427 & 5 & & 2 & 1.1760460 & 1 & & 3 & $\begin{array}{lll}5.707 & 0915\end{array}$ & 2 & & 18 & 1.2804183 & 27 & & 4 & 2.8907194 & 2 \\
\hline & 4 & $6.485 \quad 4898$ & 4 & & 7 & 1.3567864 & 6 & & 3 & $4.885 \quad 0747$ & 2 & & 4 & 1.9892809 & 2 & & 19 & 5.4333385 & 33 & & 5 & 9.7005958 & 3 \\
\hline & 5 & $1.057 \quad 2004$ & 5 & & 8 & 1.7343915 & 8 & & 4 & 1.4055788 & 2 & & 5 & 5.0446835 & 3 & & 20 & 1.4559388 & 40 & & 6 & 2.5307828 & 3 \\
\hline & 6 & 2.5045990 & 8 & & 9 & 7.1166678 & 11 & & 5 & 2.7539276 & 3 & & 6 & 9.1787613 & 4 & & 21 & 1.6552108 & 53 & & 7 & 5.0710586 & 4 \\
\hline & 7 & $7.647 \quad 1635$ & 13 & & 10 & 5.4568031 & 14 & & 6 & 3.5866647 & 4 & & 7 & 1.1762589 & 4 & & & & & & 8 & 7.7192246 & 5 \\
\hline & & & & & 11 & $2.163 \quad 2358$ & 18 & & 7 & 3.00033305 & 5 & & 8 & 1.0361473 & 5 & 23 & 0 & 2.0309579 & 1 & & 9 & 8.8053919 & 6 \\
\hline 9 & 0 & 3.2300939 & 1 & & 12 & 6.4469503 & 26 & & 8 & 1.5443189 & 6 & & 9 & 6.0765838 & 7 & & 1 & 1.7854951 & 1 & & 10 & 7.4004034 & 7 \\
\hline & 1 & $2.337 \quad 3674$ & 1 & & & & & & 9 & 4.5727586 & 8 & & 10 & $2.274 \quad I 955$ & 8 & & 2 & 1.2121472 & $i$ & & 11 & 4.4866942 & 8 \\
\hline & 2 & 8.7311640 & 2 & 14 & 0 & 2.5976616 & 1 & & 10 & $7.108 \quad 2216$ & 10 & & 11 & 5.1302689 & 10 & & 3 & 6.3379400 & 2 & & 12 & $1.911 \quad 1153$ & 9 \\
\hline & 3 & 1.6073921 & 2 & & 1 & 2.1055470 & I & & 11 & 5.0482005 & 12 & & 12 & $6.446 \quad 3349$ & 12 & & 4 & 2.5408001 & 2 & & 13 & 5.5312635 & 11 \\
\hline & 4 & 1.3308125 & 3 & & 2 & 1.1170135 & 1 & & 12 & 1.3118221 & 14 & & 13 & 4.0290598 & 14 & & 5 & 7.7595416 & 3 & & 14 & 1.0421160 & 12 \\
\hline & 5 & $4.182 \quad 1548$ & 5 & & 3 & 3.8330006 & 2 & & 13 & 8.4757895 & 18 & & 14 & 1.0566743 & 16 & & 6 & 1.7892568 & 3 & & 15 & 1.2083763 & 14 \\
\hline & 6 & 3.5643041 & 7 & & 4 & 8.3307168 & 3 & & 14 & 6.4016643 & 22 & & 15 & $8.858 \quad 2889$ & 20 & & 7 & 3.0797509 & 4 & & 16 & 7.9989178 & 17 \\
\hline & 7 & 3.6845271 & 10 & & 5 & 1,1100319 & 3 & & 15 & 9.8924570 & 28 & & 16 & 1.4808958 & 23 & & 8 & 3.8994517 & 5 & & 17 & 2.7244682 & 19 \\
\hline & 8 & 2.8114572 & 15 & & 6 & 8.6395093 & 5 & & 16 & 1.1516335 & 38 & & 17 & 1.9867421 & 28 & & 9 & $3.565 \quad 1334$ & 6 & & 18 & 4.1133833 & 22 \\
\hline & & & & & 7 & 3.6562192 & 6 & & & & & & 18 & 26951612 & 35 & & 10 & 2.2989024 & 7 & & 19 & 2.2000616 & 25 \\
\hline 10 & 0 & 3.0669310 & 1 & & 8 & 7.5458578 & 8 & 18 & 0 & 2.2936771 & $I$ & & 19 & 4.9024697 & $A_{7}$ & & 11 & 1.0146704 & 8 & & 20 & 2.9176807 & 29 \\
\hline & 1 & 2.2904565 & 1 & & 9 & 6.3818099 & 10 & & 1 & 1.9466400 & $i$ & & & & & & 12 & 2.9492001 & 10 & & 21 & 5.2097672 & 34 \\
\hline & 2 & 9.4419298 & 2 & & 10 & 1.6347825 & 12 & & 2 & $1187 \quad 8567$ & $i$ & 21 & 0 & 2.1248065 & 1 & & 13 & 5.3667858 & 12 & & 22 & 3.9340199 & 40 \\
\hline & 3 & 2.0781149 & 2 & & 11 & 6.9996012 & 16 & & 3 & 5.1829380 & 2 & & 1 & 1.8455211 & 1 & & 14 & 5.7126175 & 14 & & 23 & 9.2547548 & 49 \\
\hline & & & & & & & & & & & & & & & & & & & & & 24 & 1.6439747 & 63 \\
\hline
\end{tabular}

If $n$ is large and $c_{\nu}^{(n)}$ appreciable, the Central Limit Theorem can be used. (6) The values predicted by the Central Limit Theorem are obtained by the following replacement in Eq. (A-1):

$$
\frac{\sin x}{x} \rightarrow \exp \left(-\frac{1}{6} x^{2}\right),
$$

so that in this approximation

$$
c_{\nu}^{(n)} \approx \frac{1}{\pi} \int_{-\infty}^{\infty} d x \exp \left(-\frac{1}{6} x^{2}-2 i \nu x\right)=\sqrt{\frac{3}{\pi n}} \exp \left(-\frac{3 \nu^{2}}{n}\right)
$$


Numerical values obtained with this approximation with $n=25$ have been computed and displayed along with the correct values in Table A-II. Agreement is definitely poor for larger values of $\nu$.

Table A-II

EVALUATION OF $c_{2}^{(25)} \times 10^{9}$ BY ALTERNATIVE METHODS

\begin{tabular}{|c|c|c|c|c|c|c|c|c|}
\hline \multirow[t]{2}{*}{$\nu$} & \multicolumn{2}{|c|}{$\begin{array}{c}\text { By the } \\
\text { Longhand Method }\end{array}$} & \multicolumn{2}{|c|}{$\begin{array}{c}\text { By the Theorem of } \\
\text { Central Limits }\end{array}$} & \multicolumn{2}{|c|}{$\begin{array}{l}\text { By the Method of } \\
\text { Steepest Descent }\end{array}$} & \multicolumn{2}{|c|}{$-\frac{1}{2 n}\left[\frac{1}{8} \frac{f_{0}^{I V}}{\left(f_{0}^{\prime \prime}\right)^{2}}-\frac{5}{24} \frac{\left(f_{0}^{\prime \prime}\right)^{2}}{\left(f_{0}^{\prime \prime}\right)^{3}}\right]$} \\
\hline & & $\underline{\mathrm{q}}$ & & $\underline{q}$ & & $\underline{q}$ & & \\
\hline 0 & 1.949 & 1 & 1.954 & 1 & 1.949 & 1 & & 0.0030 \\
\hline 1 & 1.731 & 1 & 1.733 & 1 & 1.730 & 1 & & 0.0030 \\
\hline 2 & 1.212 & 1 & 1.209 & 1 & 1.210 & 1 & & 0.0030 \\
\hline 3 & 6.673 & 2 & 6.637 & 2 & 6.659 & 2 & & 0.0031 \\
\hline 4 & 2.881 & 2 & 2.865 & 2 & 2.883 & 2 & & 0.0031 \\
\hline 5 & 9.701 & 3 & 9.730 & 3 & 9.670 & 3 & & 0.0031 \\
\hline 6 & 2.531 & 3 & 2.599 & 3 & 2.527 & 3 & & 0.0032 \\
\hline 7 & 5.071 & 4 & 5.462 & 4 & 5.048 & 4 & & 0.0032 \\
\hline 8 & 7.719 & 5 & 9.029 & 5 & 7.745 & 5 & & 0.0033 \\
\hline 9 & 8.805 & 6 & 1.174 & 5 & 8.829 & 6 & & 0.0033 \\
\hline 10 & 7.400 & 7 & 1.201 & 6 & 7.419 & 7 & & 0.0034 \\
\hline 11 & 4.487 & 8 & 9.662 & 8 & 4.464 & 8 & & 0.0035 \\
\hline 12 & 1.911 & 9 & 6.115 & 9 & 1.902 & 9 & & 0.0036 \\
\hline 13 & 5.531 & 11 & 3.045 & 10 & 5.534 & 11 & & 0.0037 \\
\hline 14 & 1.042 & 12 & 1.192 & 11 & 1.041 & 12 & & 0.0038 \\
\hline 15 & 1.208 & 14 & 3.673 & 13 & 1.207 & 14 & & 0.0038 \\
\hline 16 & 7.999 & 17 & 8.902 & 15 & 7.965 & 17 & & 0.0037 \\
\hline 17 & 2.724 & 19 & 1.697 & 16 & 2.710 & 19 & & 0.0035 \\
\hline 18 & 4.113 & 22 & 2.545 & 18 & 4.096 & 22 & & 0.0031 \\
\hline 19 & 2.200 & 25 & 3.002 & 20 & 2.200 & 25 & & 0.0026 \\
\hline 20 & 2.918 & 29 & 2.785 & 22 & 2.914 & 29 & & 0.0022 \\
\hline 21 & 5.210 & 34 & 2.033 & 24 & 5.218 & 34 & & 0.0018 \\
\hline 22 & 3.934 & 40 & 1.167 & 26 & 3.930 & 40 & & 0.0017 \\
\hline 23 & 9.255 & 49 & 5.272 & 29 & 9.231 & 49 & & 0.0017 \\
\hline 24 & 1.644 & 63 & 1.873 & 31 & 1.645 & 63 & & 0.0017 \\
\hline
\end{tabular}

A better approximation procedure for the whole $x$ ange of values $\nu / \mathrm{n}$ would be the Method of Steepest Descent. By this method, the extremum of the function $2 n \ln \frac{\sin x}{x}-2 \nu x i$ is obtained, the path of integration is shifted to pass through this maximum, and the integral is evaluated under the assumption that $\nu / n$ remains constant while $n$ increases towards infinity. The extremum $\tau_{0}$ of our function is found to lie on an imaginary axis, and its position is obtained by differentiating

$$
f(\tau)=\ln \operatorname{sh} \tau-\ln \tau,
$$

and, equating the result to $\nu / \mathrm{n}$,

$$
f^{\prime}\left(\tau_{0}\right)=\operatorname{coth} \tau_{0}-\frac{1}{\tau_{0}}=\frac{\nu}{\mathrm{n}} .
$$


From this equation $\tau_{0}$ was found for every value of $\nu / n$, and $c_{\nu}^{(n)}$ was computed according to the formula

$$
\ln c_{\nu}^{(n)}=2 n f_{0}-\frac{1}{2} \ln n \pi f_{0}+\frac{1}{2 n}\left[\frac{1}{8} \frac{f_{0}^{I V}}{\left(f_{0}^{\prime \prime}\right)^{2}}-\frac{5}{24} \frac{\left(f_{0}^{n \prime \prime}\right)^{2}}{\left(f_{0}^{\prime \prime}\right)^{3}}\right]+\ldots
$$

where the values of $f$ and its derivatives have to be evaluated at $\tau=\tau_{0}$. The results of this calculation with $\mathrm{n}=25$ are also displayed in Table A-II. It is evident that this procedure gives reasonable agreement over the whole range of values of $\nu / n$. The disagreement between these approximate values and the exact values is due, at least in part, to insufficient accuracy in the determination of $\tau_{0}$ from $\mathrm{Eq}$. (A-4) (four places were used most of the time). In Table A-II we have given also the value of the last term used in Eq. (A-5). One certainly should expect the fractional error in $c(n)$ due to truncation of series Eq. (A-5) to be less than the last term used. 
Appendix B

\section{THE METHOD OF STEEPEST DESCENT AND CONVERGENCE OF MULTIPHONON EXPANSION}

For large energy values we have used formulae based upon a Taylor series expansion of $G$ about the point $t=-i / 2 k T$. This expansion gave reasonable approximation in the vicinity of $E_{-} E^{\prime} \approx \mu\left(\sqrt{E}+\sqrt{E^{\prime}}\right)^{2}$; however, the exror is considerable for other values of the ratio $E^{\prime} / E$. As we have seen in Appendix $A$, we can expect good accuracy for any ratio $E^{\prime} / E$ if we use an expansion of $G$ about a variable point $t=-i \tau$ chosen to obtain the steepest descent in the integrand. Formulae obtained by this method are difficult to evaluate numerically. But they present a clear picture of the cross section at large momentum transfers, when multiphonon expansion requires many terms.

In the method of steepest descent, we use a Taylor expansion of $G(t)$ about a variable point, $t=-i \tau$, on the imaginary axis:

$G(t)=G+G^{\prime} i(t+i \tau)-\frac{1}{2 !} G^{\prime \prime}(t+i \tau)^{2}-\frac{1}{3 !} G^{\prime \prime} i(t+i \tau)^{3}+\frac{1}{4 !} G^{i v}(t+i \tau)^{4}+\ldots$,

where coefficients

$$
G^{(n)}=\frac{d^{n}}{d \tau^{n}} \int \frac{d \omega \rho)}{\omega \operatorname{sh} \omega / 2 k T} \operatorname{ch~} \omega T
$$

are all positive. To evaluate $\sigma\left(E \rightarrow E^{\prime}, \theta\right)$, a value of $\tau$ is chosen so that the integrand in Eq. (2) is an extremum:

$$
E-E^{\prime}=\mu \gamma G^{\prime}
$$

Upon introducing a new variable of integration,

$$
x=\sqrt{\frac{1}{2} \mu \gamma G^{\prime \prime}}(t+i \tau)
$$

and expanding the integrand in Eq. (2) in powers of $\sqrt{G^{\prime \prime} / 2 \mu \gamma}$, we obtain

$$
\begin{aligned}
\int d t & \exp \left\{-\left(E-E^{\prime}\right) i\left(t+\frac{i}{2 k T}\right)+\mu \gamma[G(t)-g(0)]\right\}=\sqrt{\frac{2}{\mu \gamma G^{\prime \prime}}} \int d x \exp \\
& \left\{-\mu \gamma G^{\prime}\left(\tau-\frac{1}{2 k T}\right)+\mu \gamma[G-g(0)]-x^{2}-\sqrt{\frac{G^{\prime \prime}}{2 \mu \gamma}} \frac{2}{3} i \frac{G^{\prime \prime \prime}}{G^{\prime \prime 2}} x^{3}+\left(\frac{G \prime}{2 \mu \gamma}\right) \frac{1}{3} \frac{G^{i v}}{G^{\prime \prime 3}} x^{4}+\ldots\right\} \\
= & \sqrt{\frac{2}{\mu \gamma G^{\prime \prime}}} \exp \left\{\mu \gamma\left[-G^{\prime}\left(\tau-\frac{1}{2 k T}\right)+G-g(0)\right]\right\} \cdot \int d x e^{-x^{2}} \\
& \left\{1-\sqrt{\frac{G^{\prime \prime}}{2 \mu \gamma}} \frac{2}{3} i \frac{G^{\prime \prime \prime}}{G^{\prime \prime 2}} x^{3}+\left(\frac{G \prime}{2 \mu \gamma}\right)\left[\frac{1}{3} \frac{G^{i v}}{G^{\prime \prime 3}} x^{4}-\frac{2}{9} \frac{G^{\prime \prime 2}}{G^{\prime \prime 4}} x^{6}\right]+\ldots\right\} .
\end{aligned}
$$


Thus, after integration, we have

$$
\begin{aligned}
\sigma\left(E \rightarrow E^{\gamma}, \theta\right)= & \left(\sigma_{b} / 8 \pi^{2}\right)\left(E^{\prime} / E\right)^{1 / 2}\left(4 \pi / 2 \mu \gamma G^{10}\right)^{1 / 2} \\
& \cdot \exp \left\{\mu \gamma\left[-G^{\prime}\left(\tau-\frac{1}{2 k T}\right)+G-g(0)\right]\right\} \\
& \cdot\left\{1+\frac{G^{n}}{2 \mu \gamma}\left[\frac{3}{12} \frac{G^{i v}}{G^{183}}-\frac{5}{12} \frac{G^{182}}{G^{184}}\right]+\ldots\right\},
\end{aligned}
$$

a convenient expression for large momentum transfers when multiphonon expansion becomes impractical.

By contemplation of Eq. (B-3) we can make a judgment on the number of phonons necessary to obtain the differential cross section. It is reasonable to expect that, when $E q .(B-3)$ is approximately valid, this number is roughly equal to the number of terms required in the power series expansion of $\exp (\mu \gamma \mathrm{G})$. Thus the largest contribution is expected for $n=\mu \gamma \mathrm{G}$. Since

$$
\frac{\mathrm{d}^{2}}{\mathrm{dn} \mathrm{n}^{2}} \ln \frac{1}{\mathrm{n} !}(\mu \gamma \mathrm{G})^{\mathrm{n}} \approx-\frac{1}{\mu \gamma \mathrm{G}}
$$

one would obtain the value of the exponential within about two per cent if one uses

$$
\mathrm{n}_{\max }=(\sqrt{\mu \gamma \mathrm{G}}+1)^{2}
$$

Actually, applying the method of steepest descent to each term of the phonon expansion we see that the "half-width" is somewhat smaller and that

$$
\mathrm{n}_{\max }=\left[\sqrt{\mu \gamma \mathrm{G}}+\sqrt{1-\left(\mathrm{G}^{2} / \mathrm{GG}^{n}\right)}\right]^{2}
$$

would be satisfactory. Thus, for large $\mu \gamma \mathrm{G}$ only comparatively small number of phonons at the end of expansion contribute significantly towards the sum. The second term in Eq. $(B-3)$ is then

$$
\frac{1}{12} \frac{1}{\mu \gamma G} \frac{3 G^{i v} G^{n}-5 G^{n 12}}{2 G^{13}} G \approx \frac{1}{12 n_{\max }} \frac{3 G^{i v} G^{n 1}-5 G^{n 82}}{2 G^{n 3}} G
$$

If $T$ (and the ratio $|E-E| / \mu \gamma$ ) is very large, this term is approximately equal to $-1 / 12 n_{\text {max }}$ and $\mathrm{Eq} .(B-3)$ joins quite smoothly our expansion of $n_{\max }(=25)$ phonons. However, for smaller $\tau$ this term can be considerably larger in absolute value. In such cases, one could try to approximate every multiphonon term by a Gaussian (or modified Gaussian) distribution. And, indeed, one can demonstrate(2) that such an approximation is good for individual terms. However, the number of terms required for evaluation of $\sigma\left(E \rightarrow E^{\natural}, \theta\right)$ 
is laxge. And, since the Gaussian distribution depends only on the second derivative of $G$, it cannot be depended upon to yield correctly the second term of Eq. (B-3), which requires knowledge of higher derivatives. Thus, at present we remain with the unpleasant need to evaluate exactly many terms in multiphonon expansion in some cases (as for graphite at high temperatures) if we want to join smoothly the method of steepest descent to the multiphonon expansion.

In evaluation of $\sigma\left(E \rightarrow E^{1}\right)$ we encounter also both multiphonon expansion and an asymptotic expression. Multiphonon expansion here needs to be used also at very high initial energies if energy loss is not large. When $|E-E|$ is fixed finite and $\mu\left(\sqrt{E}+\sqrt{E^{\prime}}\right)^{2}$ keeps increasing, we can no longer nelgect the second term in Eq. (26). (The asymptotic expansion for it does not "converge.") Indeed the appropriate procedure for such a case would be to neglect the first term, since $\mu\left(\sqrt{\mathrm{E}}+\sqrt{\mathrm{E}^{\prime}}\right)^{2}$ is large and $[G(t)-g(0)]$ is negative. Since

$$
\mu\left(\sqrt{E}-\sqrt{E^{1}}\right)^{2}=\mu\left(E-E^{1}\right)^{2} /\left(\sqrt{E}+\sqrt{E^{1}}\right)^{2}
$$

is small when $|E-E|$ is finite and $\left(\sqrt{E}+\sqrt{E^{2}}\right)^{2}$ is large, we can expand our integrand in a power series in $\mu$ :

$$
\begin{aligned}
=\frac{\exp \left\{\mu\left(\sqrt{E}-\sqrt{E^{\prime}}\right)^{2}[G(t)-g(0)]\right\}}{G(t)-g(0)}= & -\frac{1}{G(t)-g(0)}-\frac{\mu\left(E-E^{3}\right)^{2}}{\left(\sqrt{E}+\sqrt{\left.E^{3}\right)^{2}}\right.} \\
& -\frac{1}{2} \frac{\mu^{2}\left(E-E^{3}\right)^{4}}{\left(\sqrt{E}+\sqrt{E^{1}}\right)^{4}}[G(t)-g(0)]-\ldots
\end{aligned}
$$

and integrate term by term. Fourier transformation of

$$
\frac{1}{g(0)-G(t)}-\frac{1}{g(0)}
$$

will now give the main inelastic contribution. Thus, the inelastic cross section is approximately equal to

$$
\begin{aligned}
\sigma\left(E \rightarrow E^{\prime}\right) & \approx\left(\sigma_{b} / 8 \pi \mu E\right) \int d t \exp -\left(E-E^{\prime}\right)\left(t+\frac{i}{2 k T}\right)\left[\frac{1}{g(0)-G(t)}-g(0)\right] \\
& =\left(\sigma_{b} / 8 \pi \mu E\right) \frac{1}{g(0)} \cdot \int d t \exp \left[-\left(E-E^{\prime}\right)\left(t+\frac{i}{2 k T}\right)\right] \sum_{n=1}^{\infty}\left[\frac{G(t)}{g(0)}\right]^{2} \ldots
\end{aligned}
$$

Since the nearest zero of $g(0)-G(t)$ is located at $t=-i / 2 k T$, for large values of $\left(E-E^{\prime}\right), E q .(B-6)$ gives correct value for the inelastic cross section: $\left(\sigma_{\mathrm{b}} / 4 \mu \mathrm{E}\right)$. The same value, of course, is obtained also from Eq. (30) when $\mu\left(\sqrt{E}+\sqrt{E^{1}}\right)^{2}$ is large and $\eta$ is large negative. 
Applying the method of steepest descent to each term of Eq. $(B-6)$, we see that the largest term, $S_{n}$, is obtained for $n=g(0)\left|E-E^{n}\right|$. If $n$ is large,

$$
\frac{d^{2}}{d n^{2}} \ln S_{n} \approx-\frac{1}{g(0)|E-E|} \frac{1}{a_{2} g(0)-1}
$$

Thus

$$
\mathrm{n}_{\max }=\left(\sqrt{\left|E-E^{i}\right| g(0)}+\sqrt{a_{2} g(0)-1}\right)^{2}
$$

terms should be satisfactory for the evaluation of Eq. (B-6). When the first term in Eq. $(B-7)$ becomes smaller than the second, the number of phonons given by $\mathrm{Eq} .(\mathrm{B}-7)$ is insufficient. It seems that one needs about $4\left[\mathrm{a}_{2} g(0)-1\right]$ terms even for small energy loss. Moreover, we believe also that for $\left|E-E^{p}\right| \geq a_{2} E q .(B-6)$ will have approached its limiting value. Thus, if we use $n_{\max } \gtrsim 4 a_{2} g(0)$, we should have a fairly smooth transition between multiphonon expansion and the asymptotic expression. 


\section{Appendix C}

\section{AVERAGING OVER DIRECTIONS OF POLARIZATION FOR GRAPHITE}

For calculation of the scattering cross section from polycrystalline graphite $\rho$ used in the initial formula, Eq. (2), can be represented as an interpolation:

$$
\rho=\rho_{1} \ell^{2}+\rho_{2}\left(1-\ell^{2}\right)
$$

between frequency distribution perpendicular to the planes of crystal lattice, $\rho_{1}$, and frequency distribution in the planes, $\rho_{2} .(3)$ The scattering cross section then is obtained upon integration of the final results for cross section over the directions of lattice orientation, $0 \leq \ell \leq 1$. Actually, in Chaptex II, calculations of $f_{j}^{(1)}, g(0), a_{2}$, and $a_{3}$ are performed separately for both sets of values $\rho_{1 j}$ and $\rho_{2 j}$, and a common scaling factor is determined. Then, for every needed value of $l$ appropriate quantities $f_{j}^{(1)}, g(0), a_{2}$, and $a_{3}$ are determined by an interpolation procedure, Eq. $(\mathrm{C}-1)$.

Since evaluation of the cross section is a quite elaborate and long process, we have chosen a Gaussian $(11,12)$ integration process. We notice here that our integrand is an even function of $l$. Thus, if we would expand the limits of integration from -1 to +1 , we would not need actually to calculate the values of the integrand for negative values of $l$. Thus (considering only Gaussian integration schemes with even numbers of values for $\ell)$, we see that by actually calculating the integrand value at $\mathrm{n}$ points we approximate the integrand with a polynomial of degree $4 \mathrm{n}-1$. (Or, we can say that we approximate our integrand with a polynomial which coincides with the integrand at $3 n$ points, of which 2 n are chosen arbitrarily.) We can see easily that this integration scheme is exact for a Placzek expansion (in powers of $\mu$ ) that neglects terms with $\mu^{2 n}$ and higher powers. It is also exact for expansion of $S$ in a power sexies of $\alpha$ up to and including the term with $\alpha^{2 n-1}$. These considerations lead us to believe that only a few points are needed for quite satisfactory integration over $l$. Indeed, in several previous calculations graphite has been approximated by a cubic crystal, using only the total frequency spectrum, and thus essentially using only one point in our Gaussian integration scheme. Upon contemplating the increase of accuracy obtained by using the Placzek expansion, we believe that the additional labor required in using at least two points is well justified. The values of $l$ and corresponding weighting coefficients (13) have been given in Table C-I. 
Table C-I

CONSTANTS FOR GAUSSIAN INTEGRATION OF AN EVEN FUNCTION

\begin{tabular}{|c|c|c|}
\hline $\mathrm{n}$ & $\ell_{\mathrm{i}}^{(\mathrm{n})}$ & $\mathrm{W}_{\mathrm{i}}^{(\mathrm{n})}$ \\
\hline 1 & 0.57735027 & 1.00000000 \\
\hline & 0.33998104 & 0.65214515 \\
& 0.86113631 & 0.34785485 \\
& 0.23861919 & 0.46791393 \\
4 & 0.66120939 & 0.36076157 \\
& 0.93246951 & 0.17132449 \\
& 0.18343464 & 0.36268378 \\
& 0.52553241 & 0.31370665 \\
& 0.79666648 & 0.22238103 \\
& 0.96028986 & 0.10122854 \\
& 0.14887434 & 0.29552422 \\
& 0.43339539 & 0.26926672 \\
& 0.67940957 & 0.21908636 \\
& 0.86506337 & 0.14945135 \\
& 0.97390653 & 0.06667134 \\
\hline
\end{tabular}




\section{ACKNOWLEDGMENTS}

The author is grateful to Professor R. Courant, Dr. E. H. Bareiss and $\mathrm{Mr} . \mathrm{H}$. Greenspan for several stimulating discussions. He is also indebted to Mr. L. C. Kvitek whose very tedious numerical calculations are reported in Appendix A.

\section{REFERENCES}

1. V.Z. Jankus, Numerical Evaluation of the Neutron Scattering Cross Section in the Incoherent Approximation, Trans. Am. Nuclear Soc., 3, $269(1960)$.

2. P. Schofield and A. Hassit, The Calculation of Thermal Neutron Spectra, Proceedings of the Second United Nations International Conference on the Peaceful Uses of Atomic Energy, Geneva, Switzerland, $16,217$.

3. Alf Sjölander, Multiphonon Processes in Slow Neutron Scattering by Crystals, Ark. Fys., 14, 315 (1958).

4. Donald E. Parks, Relation of Crystal Symmetry in Graphite to Lattice Vibrations and Their Interaction with Slow Neutrons, GA-2125 (1961).

5. R. J. Glauber, Time-dependent Displacement Correlations and Inelastic Scattering by Crystals, Phys. Rev., 98, 1692 (1955).

6. H. Cramer, Mathematical Methods of Statistics, Princeton U. Press, Princeton, N. J (1946) p. 213.

7. P.A. Egelstaff, The Treatment of Thermal Neutron Scattering Law Data, AERE-R 3622 (1961).

8. G. Placzek, Incoherent Neutron Scattering by Polycrystals, Phys. Rev., 93, 895 (1954).

9. W. Grobner and N. Hofreiter, Integraltafel, Zweiter Teil, SpringerVerlag, Vienna (1950), 314,8b; p. 66.

10. Erdelyi Magnus Oberhettinger Tricomi, Tables of Integral Transforms, Vol. I, McGraw-Hill Book Co., Inc., New York (1954) p. 20.

11. C. F. Gauss, Methodus Nova Integralium Valores per Approximationem Inveniendi, Göett. Comm., Vol. 3 (1814); or Werke, Vol. 3 , p. 163 .

12. W. E. Milne, Numerical Calculus, Princeton University Press, Princeton, N. J. (1949) p. 285.

13. A. N. Lowan, N. Davids, and A. Levenson, Table of the Zeros of the Legendre Polynomials of Order $1-16$ and Weight Coefficients for Gauss Mechanical Quadrature Formula, Am. Math. Soc. Bul., 48, 739 (1942). 